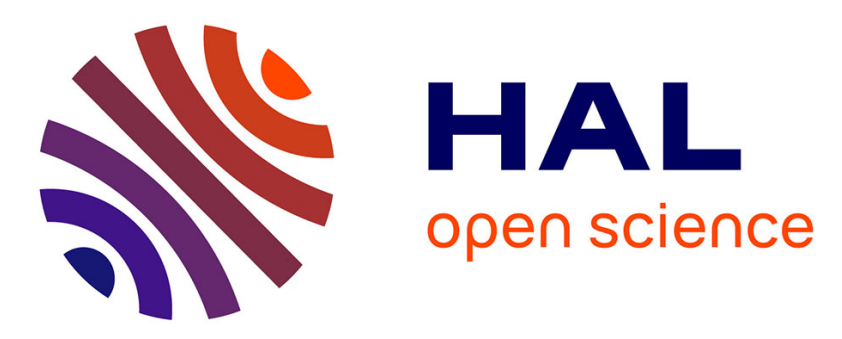

\title{
A variant of the Hardy-Ramanujan theorem
}

\author{
M. Ram Murty, V Kumar Murty
}

\section{To cite this version:}

M. Ram Murty, V Kumar Murty. A variant of the Hardy-Ramanujan theorem. Hardy-Ramanujan Journal, 2022, Special Commemorative volume in honour of Srinivasa Ramanujan - 2021, Volume 44 - Special Commemorative volume in honour of Srinivasa Ramanujan - 2021, pp.32 - 40. 10.46298/hrj.2022.8343 . hal-03251106v2

\section{HAL Id: hal-03251106 https://hal.science/hal-03251106v2}

Submitted on 20 Dec 2021

HAL is a multi-disciplinary open access archive for the deposit and dissemination of scientific research documents, whether they are published or not. The documents may come from teaching and research institutions in France or abroad, or from public or private research centers.
L'archive ouverte pluridisciplinaire HAL, est destinée au dépôt et à la diffusion de documents scientifiques de niveau recherche, publiés ou non, émanant des établissements d'enseignement et de recherche français ou étrangers, des laboratoires publics ou privés. 


\title{
A variant of the Hardy-Ramanujan theorem
}

\author{
M. Ram Murty and V. Kumar Murty*
}

Dedicated to the memory of Srinivasa Ramanujan

\begin{abstract}
For each natural number $n$, we define $\omega^{*}(n)$ to be the number of primes $p$ such that $p-1$ divides $n$. We show that in contrast to the Hardy-Ramanujan theorem which asserts that the number $\omega(n)$ of prime divisors of $n$ has a normal order $\log \log n$, the function $\omega^{*}(n)$ does not have a normal order. We conjecture that for some positive constant $C$,

$$
\sum_{n \leq x} \omega^{*}(n)^{2} \sim C x(\log x)
$$

Another conjecture related to this function emerges, which seems to be of independent interest. More precisely, we conjecture that for some constant $C>0$, as $x \rightarrow \infty$,

$$
\sum_{[p-1, q-1] \leq x} \frac{1}{[p-1, q-1]} \sim C \log x
$$

where the summation is over primes $p, q \leq x$ such that the least common multiple $[p-1, q-1]$ is less than or equal to $x$. Keywords. prime divisors, normal order, Brun's sieve, Brun-Titchmarsh inequality.

2010 Mathematics Subject Classification. Primary 11N25, 11N36. Secondary 11N37, 11N64.
\end{abstract}

\section{Introduction}

Let $\omega(n)$ be the number of distinct prime divisors of $n$. In 1917, Hardy and Ramanujan [HR1917] showed that $\omega(n)$ has normal order $\log \log n$. They proved that for any $\epsilon>0$, the number of $n \leq x$ such that

$$
|\omega(n)-\log \log n|>(\log \log n)^{\frac{1}{2}+\epsilon}
$$

is $\mathbf{o}(x)$ as $x \rightarrow \infty$. In this article, we study the function $\omega^{*}(n)$ which counts the number of primes $p$ for which $p-1$ divides $n$. This function appears in numerous places, including in questions related to primality testing. For instance, in 1899, Korselt showed that $n \mid a^{n}-a$ for every $a$ if and only if $(p-1) \mid(n-1)$ for every prime $p$ divisor of $n$. In 1910, R.D. Carmichael noted that $n=561=3 \cdot 11 \cdot 17$ satisfies this condition and thus gives a concrete counterexample to the converse of Fermat's little theorem. One might at first suspect that it would behave similarly to $\omega(n)$ but in fact it seems to be rather different. In particular, we show here that it does not have a normal order.

Theorem 1.1. The average order of $\omega^{*}(n)$ is $\log \log n$ while the average order of $\omega^{*}(n)^{2}$ is at least $C(\log \log n)^{3}$ for a suitable constant $C$. More precisely, we have

$$
\sum_{n \leq x} \omega^{*}(n)=x(\log \log x)+B x+\mathbf{O}(x / \log x),
$$

while

$$
\sum_{n \leq x} \omega^{*}(n)^{2} \geq C x(\log \log x)^{3}
$$

for a suitable constant $C>0$.

${ }^{*}$ The research of both authors was partially supported by NSERC Discovery Grants. We thank episciences.org for providing open access hosting of the electronic journal Hardy-Ramanujan Journal 
Prachar [Pra55] proved (1.1) in 1955. His paper may have been the impetus for his joint paper with Erdös [EP55] that appeared in the same journal, where they show using Brun's sieve that the number of pairs of primes $p, q$ such that the least common multiple $[p-1, q-1]$ is less than $x$ is $\mathbf{O}(x \log \log x)$.

Since $\omega^{*}(n)$ is really the number of divisors $d$ of $n$ such that $d+1$ is prime, and by the prime number theorem, the probability that $d+1$ is prime is $1 / \log (d+1)$, heuristic considerations would suggest that

$$
\omega^{*}(n) \asymp \sum_{d \mid n} \frac{1}{\log (d+1)} \asymp \frac{d(n)}{\log n},
$$

where $d(n)$ is the number of divisors of $n$. This heuristic is certainly consistent with our result on the average order of $\omega^{*}(n)$. If this heuristic were true (at least on average), we may conjecture that for some constant $C>0$,

$$
\sum_{n \leq x} \omega^{*}(n)^{2}=C x \log x+\mathbf{O}(x)
$$

since (see for example, page 62 of [Ram08]),

$$
\sum_{n \leq x} d(n)^{2}=\frac{x}{\pi^{2}}(\log x)^{3}+\mathbf{O}\left(x \log ^{2} x\right) .
$$

In fact, Ramanujan discovered the more precise asymptotic formula [Ra1916]:

$$
\sum_{n \leq x} d(n)^{2}=\frac{x}{\pi^{2}}(\log x)^{3}+\left(\frac{12 \gamma-3}{\pi^{2}}-\frac{36}{\pi^{4}} \zeta^{\prime}(2)\right) x(\log x)^{2}+A x \log x+B x+\mathbf{O}\left(x^{3 / 5+\epsilon}\right),
$$

for certain "complicated constants" $A, B$ and any $\epsilon>0$. He remarks in a footnote that if we assume the Riemann hypothesis, the error can be improved to $\mathbf{O}\left(x^{1 / 2+\epsilon}\right)$.

In [Pra55], Prachar shows that

$$
\sum_{n \leq x} \omega^{*}(n)^{2}=\mathbf{O}\left(x \log ^{2} x\right),
$$

using Brun's sieve. This does not preclude the possibility that (1.3) still holds.

Indeed, we will improve Prachar's theorem and progress towards conjecture (1.3) through the following theorem.

\section{Theorem 1.2.}

$$
\sum_{n \leq x} \omega^{*}(n)^{2}=\mathbf{O}(x \log x) .
$$

In the paper alluded to above, Prachar [Pra55] shows assuming the generalized Riemann hypothesis for Dirichlet $L$-functions, that for infinitely many $n$,

$$
\omega^{*}(n)>\exp \left((\log \sqrt{2}-\epsilon) \frac{\log n}{\log \log n}\right) .
$$

Unconditonally, he shows that for some constant $c>0$,

$$
\omega^{*}(n)>\exp \left(\frac{c \log n}{(\log \log n)^{2}}\right),
$$

for infinitely many $n$. However, by a slight re-arrangement of Prachar's proof and a minor modification, Adleman, Rumely and Pomerance (see Proposition 10 in [APR83]) showed that for some constant $c>0$,

$$
\omega^{*}(n)>\exp \left(\frac{c \log n}{\log \log n}\right),
$$


for infinitely many squarefree $n$ without any hypothesis. By contrast, Ramanujan [Ra1915] showed in his celebrated paper "Highly composite numbers" that for all $n \geq 2$,

$$
\omega(n) \leq \frac{\log n}{\log \log n}+\mathbf{O}\left(\frac{\log n}{(\log \log n)^{2}}\right) .
$$

From this medley of results, we therefore see that the behaviour of $\omega^{*}(n)$ is drastically different from $\omega(n)$.

\section{Preliminary lemmas}

We record here several results that will be used in proving our main results.

Lemma 2.1. Let $d$ be a natural number. Let $p(d, a)$ be the smallest prime $p \equiv a(\bmod d)$. Then

$$
\sum_{\substack{p \leq x \\ p \equiv a(\bmod d)}} \frac{1}{p}=\frac{1}{\phi(d)} \log \log x+\frac{1}{p(d, a)}+\mathbf{O}\left(\frac{\log d}{\phi(d)}\right)
$$

uniformly for $d \leq x$. In particular,

$$
\sum_{\substack{p \leq x \\ p \equiv 1(\bmod d)}} \frac{1}{p}=\frac{1}{\phi(d)} \log \log x+\mathbf{O}\left(\frac{\log d}{\phi(d)}\right)
$$

This is due to Norton and appears in [Nor76] as Lemma 6.3. The form we have stated appears also in [Pom77] where it is a remark after Theorem 1. The second statement of the lemma follows on noting that $p(d, 1) \geq d$. Weaker versions of this theorem were used earlier by Erdös [Erd48] and Murty-Murty [MM79], [MM84].

Lemma 2.2. The number of pairs of primes $p, q$ such that the lcm $[p-1, q-1] \leq x$ is $\mathbf{O}(x)$.

This is essentially due to Erdös and Prachar [EP55] and what they actually prove using Brun's sieve is the estimate $\mathbf{O}(x \log \log x)$. However, in a footnote to their paper, they remark that the number of solutions is $\mathbf{O}(x)$. As we need this result, and the exposition in [EP55] is far from satisfactory, we give a short proof in the next section. Strangely, this paper appears only the authorship of Erdös in Mathematical Reviews.

Lemma 2.3. (The Brun-Titchmarsh inequality) Let $d$ be a natural number and let $(a, d)=1$. Let $\pi(x, d, a)$ be the number of primes $p \leq x$ with $p \equiv a(\bmod d)$. Then

$$
\pi(x, d, a) \leq \frac{2 x}{\phi(d) \log (x / d)}, \quad d<x .
$$

This form of the Brun-Titchmarsh inequality is due to Montgomery and Vaughan [MV73] and represents a culmination of a series of results beginning with Brun, then Titchmarsh, and a host of other mathematicians who saw the need for such an inequality in many problems of analytic number theory. The following establishes an estimate that goes beyond the range of Lemma 2.1.

Lemma 2.4. For $x>d$,

$$
\sum_{\substack{p \leq x \\ p \equiv a(\bmod d)}} \frac{1}{p} \ll \frac{1}{p(d, a)}+\frac{\log \log (x / d)}{\phi(d)} .
$$

In particular, if $a=1$, we have

$$
\sum_{\substack{p \leq x \\ p \equiv 1(\bmod d)}} \frac{1}{p} \ll \frac{\log \log x}{\phi(d)} .
$$


Proof. This is an application of partial summation and the Brun-Titchmarsh inequality (Lemma 2.3). We see that

$$
\sum_{\substack{2 d<p \leq x \\ p \equiv a(\bmod d)}} \frac{1}{p} \ll \frac{\pi(x, d, a)}{x}+\int_{2 d}^{x} \frac{\pi(t, d, a)}{t^{2}} d t .
$$

By Lemma 2.3, the integral is

$$
\ll \int_{2 d}^{x} \frac{d t}{\phi(d) t(\log t-\log d)} .
$$

After a change of variable and integration, and including the (possible) primes $p \leq 2 d$ with $p \equiv$ $a(\bmod d)$, the result follows.

Lemma 2.5. Let $a, b$ be coprime natural numbers such that $a b(a-b) \neq 0$. The number of natural numbers $n \leq x$ such that both an +1 and bn +1 are prime is

$$
\ll \frac{x}{(\log x)^{2}} \prod_{p \mid a b(a-b)}\left(1+\frac{1}{p}\right),
$$

where the implied constant is absolute.

Proof. This is a consequence of Brun's sieve, the details of which can be found in [Hal74]. In particular, we apply Theorem 2.3 in [Hal74] to our context.

Lemma 2.6. For any natural number $d$, and $a \geq 1$, we have

$$
\sum_{d \leq n \leq x, n \equiv a(\bmod d)} \frac{1}{n} \ll \frac{\log (2 x / d)}{d} .
$$

Proof. We have

$$
\sum_{d \leq n \leq x, n \equiv a(\bmod d)} \frac{1}{n} \leq \sum_{t \leq x / d} \frac{1}{d t+a} \leq \sum_{t \leq x / d} \frac{1}{d t} \ll \frac{\log (2 x / d)}{d},
$$

by elementary calculus.

\section{Lemma 2.7 .}

$$
\sum_{1<a, b \leq x^{1 / 4},(a, b)=1} \frac{1}{a b} \prod_{p \mid a b(a-b)}\left(1+\frac{1}{p}\right) \ll(\log x)^{2} .
$$

Proof. Noting that

$$
\prod_{p \mid n}\left(1+\frac{1}{p}\right)=\sum_{d \mid n} \frac{\mu^{2}(d)}{d}
$$

we have that our sum is bounded by

$$
\sum_{d \leq x^{3 / 4}} \frac{\mu^{2}(d)}{d} \sum_{a, b \leq x^{1 / 2}, d \mid a b(a-b)}^{\prime} \frac{1}{a b},
$$

where the dash on the sum means we sum over $a, b$ with $a \neq b$. By the Chinese remainder theorem, we see that $d \mid a b(a-b)$ means that for each prime divisor $p$ of $a b(a-b)$, we must have $a \equiv 0$ or $b \equiv 0$ or $a \equiv b(\bmod p)$. Thus, for each prime $p$, there are at most $3 p$ pairs $(a, b)(\bmod p)$ for which $p \mid a b(a-b)$. Modulo $d$, there are at most $3^{\omega(d)} d$ such pairs. We partition the inner sum over each of these residue classes $(\bmod d)$ and by symmetry, we may suppose that in the sum, $a<b$. Splitting this sum into 
$b \leq d$ and $b>d$, we see the inner sum is at most $\log \left(3^{\omega(d)} d\right) \ll \log d$ and the total contribution to our sum in question is

$$
\ll \sum_{d \leq x^{3 / 4}} \frac{\mu^{2}(d) \log d}{d} \ll(\log x)^{2} .
$$

For the second part, we apply Lemma 2.6 and see that the inner sum is at most

$$
3^{\omega(d)} d\left(\frac{\log 2 x}{d}\right)^{2} \ll \frac{3^{\omega(d)}(\log 2 x)^{2}}{d}
$$

and inserting this into our sum, we find the final contribution is $\ll(\log x)^{2}$ because the series

$$
\sum_{d=1}^{\infty} \frac{\mu^{2}(d) 3^{\omega(d)}}{d^{2}}
$$

converges. This completes the proof.

\section{The Erdös-Prachar theorem revisited}

We give below, in a clear and concise way, a proof of the theorem of Erdös and Prachar recorded in Lemma 2.2. We want to count the number of primes pairs $p, q$ such that $[p-1, q-1] \leq x$. For each $d \leq x$, let us first count the number $N_{d}$ of prime pairs $p, q$ such that the gcd $(p-1, q-1)=d$ and $[p-1, q-1] \leq x$. This is bounded by the number of prime pairs $p, q$ such that $p \equiv q \equiv 1(\bmod d)$ and $(p-1)(q-1) \leq d x$. Without any loss of generality, we may suppose $p \leq q$ so that $p \leq 2 \sqrt{d x}$. Thus,

$$
N_{d} \leq 2 \sum_{\substack{p \leq 2 \sqrt{d x} \\ p \equiv 1(\bmod d)}} \pi(2 d x / p, d, 1) .
$$

We want to apply the Brun-Titchmarsh inequality but to do so, must ensure $p$ is not too close to $x$. This motivates the consideration of two cases: $d \leq x^{3 / 4}$ and $d>x^{3 / 4}$. In the first case, we see that $p \leq 2 \sqrt{d x}$ implies $p \leq x^{7 / 8}$. Thus, by the Brun-Titchmarsh inequality (Lemma 2.3),

$$
N_{d} \ll \sum_{\substack{p \leq \sqrt{d x} \\ p \equiv 1(\bmod d)}} \frac{x}{p} \frac{d}{\phi(d) \log x}
$$

Noting that $\sqrt{d x}>d$, we get by Lemma 2.4 ,

$$
N_{d} \ll \frac{d x \log \log x}{\phi(d)^{2} \log x} .
$$

Summing this over $d \leq x^{3 / 4}$ and using the elementary estimate

$$
\sum_{d<z} \frac{d}{\phi(d)^{2}} \ll \log z,
$$

we get in the first case, a final estimate of $\mathbf{O}(x \log \log x)$.

In the second case, $x \geq d>x^{3 / 4}$. We proceed to estimate $N_{d}$. We write $p-1=a d$ and $q-1=b d$ with $(a, b)=1$ and observe that as $x \geq[p-1, q-1]=a b d$, we have $a b \leq x / d$ which implies $a b \leq x^{1 / 4}$. By Lemma 2.5, the number of $d \leq x / a b$ such that both $a d+1$ and $b d+1$ are prime is

$$
\ll \frac{x}{a b(\log x)^{2}} \prod_{p \mid a b(a-b)}\left(1+\frac{1}{p}\right) .
$$


We sum this over $a, b \leq x^{1 / 4}$ and apply Lemma 2.7 to deduce the result that the estimate for this range of $d$ is $\mathbf{O}(x)$.

This is, in essence, what is contained in the core of the paper [EP55]. In a footnote, the authors state that the result can be improved to $\mathbf{O}(x)$ using a result of Titchmarsh. As no details are given, we present them now. By our account above, we need to refine the estimate for the first case, when $d<x^{3 / 4}$. Looking at (3.5), the sum to estimate is

$$
\frac{x}{\log x} \sum_{p \leq x} \frac{A_{p}}{p}
$$

where

$$
A_{p}=\sum_{d \mid p-1, d<x^{3 / 4}} \frac{d}{\phi(d)} .
$$

Now by the Brun-Titchmarsh inequality,

$$
\sum_{p \leq x} A_{p}=\sum_{d<x^{3 / 4}} \frac{d}{\phi(d)} \pi(x, d, 1) \ll \sum_{d<x^{3 / 4}} \frac{d}{\phi(d)^{2}} \frac{x}{\log x} \ll \frac{x}{\log x} \sum_{d<x^{3 / 4}} \frac{d}{\phi(d)^{2}} \ll x,
$$

where in the last step, we applied the elementary estimate

$$
\sum_{d<x} \frac{d}{\phi(d)^{2}} \ll \log x
$$

With (3.7), in hand, we now apply dyadic subdivision to the sum appearing in (3.6):

$$
\sum_{p \leq x} \frac{A_{p}}{p} \ll \sum_{k=1}^{\log x} \sum_{2^{k} \leq p<2^{k+1}} \frac{A_{p}}{p} \ll \sum_{k=1}^{\log x} \frac{1}{2^{k}}\left(\sum_{p<2^{k+1}} A_{p}\right) \ll \log x,
$$

which completes the proof.

\section{4. $\quad$ Proof of Theorem 1.1}

The average order is easily seen to be

$$
\sum_{n \leq x} \omega^{*}(n)=\sum_{p \leq x}\left[\frac{x}{p-1}\right]=x(\log \log x)+B x+\mathbf{O}(x / \log x)
$$

using Mertens theorem. As mentioned earlier, this was also observed by Prachar [Pra55].

On the other hand, we have

$$
\sum_{n \leq x} \omega^{*}(n)^{2}=\sum_{n \leq x}\left(\sum_{(p-1) \mid n} 1\right)^{2}=\sum_{p, q \leq x}\left[\frac{x}{[p-1, q-1]}\right]
$$

where $[p-1, q-1]$ denotes the least common multiple of $p-1$ and $q-1$ and the sum is over primes $p, q \leq x$. Using Lemma 2.2, we have

$$
\sum_{n \leq x} \omega^{*}(n)^{2}=\sum_{p, q \leq x}\left[\frac{x}{[p-1, q-1]}\right]=\sum_{p, q \leq x} \frac{x}{[p-1, q-1]}+\mathbf{O}(x) .
$$


Separating the terms with $p=q$ and $p \neq q$, and using the relation

$$
[p-1, q-1]=(p-1)(q-1) /(p-1, q-1)
$$

between the least common multiple and the greatest common divisor, we deduce that

$$
\sum_{n \leq x} \omega^{*}(n)^{2}=\sum_{\substack{p, q \leq x \\ p \neq q}} \frac{x}{(p-1)(q-1)}(p-1, q-1)+\mathbf{O}(x) .
$$

Allowing for $p=q$ in the sum and estimating the error, we see that

$$
\sum_{n \leq x} \omega^{*}(n)^{2}=\sum_{p, q \leq x} \frac{x}{(p-1)(q-1)}(p-1, q-1)+\mathbf{O}(x) .
$$

Now,

$$
(p-1, q-1)=\sum_{d|(p-1), d|(q-1)} \phi(d) .
$$

Inserting (4.9) into (4.8), we get

$$
\sum_{n \leq x} \omega^{*}(n)^{2}=x \sum_{d \leq x} \phi(d)\left(\sum_{\substack{p \leq x \\ p \equiv 1 \bmod d}} \frac{1}{p-1}\right)^{2}+\mathbf{O}(x) .
$$

We get a lower bound if we restrict our sum to $d \leq z$ with $z$ to be chosen suitably later. We apply Lemma 2.1 to get

$$
\sum_{n \leq x} \omega^{*}(n)^{2} \geq x \sum_{d \leq z} \phi(d)\left\{\frac{1}{\phi(d)} \log \log x+\mathbf{O}\left(\frac{\log d}{\phi(d)}\right)\right\}^{2}+\mathbf{O}(x \log \log x) .
$$

Simplifying, we see that the right hand side is

$$
x(\log \log x)^{2} \sum_{d \leq z} \frac{1}{\phi(d)}+\mathbf{O}\left(x(\log \log x) \sum_{d \leq z} \frac{\log d}{\phi(d)}\right)+\mathbf{O}\left(x \sum_{d \leq z} \frac{(\log d)^{2}}{\phi(d)}\right) .
$$

By elementary number theory, we have the following:

$$
\begin{gathered}
\sum_{d \leq z} \frac{1}{\phi(d)}=A \log z+\mathbf{O}(1) \\
\sum_{d \leq z} \frac{\log d}{\phi(d)}=\mathbf{O}\left((\log z)^{2}\right)
\end{gathered}
$$

and

$$
\sum_{d \leq z} \frac{(\log d)^{2}}{\phi(d)}=\mathbf{O}\left((\log z)^{3}\right)
$$

We choose $z=(\log x)^{c}$. Thus, our final inequality is

$$
\sum_{n \leq x} \omega^{*}(n)^{2} \geq A c x(\log \log x)^{3}+\mathbf{O}\left(c^{2} x(\log \log x)^{3}\right)
$$

and this gives upon choosing $c$ sufficiently small,

$$
\sum_{n \leq x} \omega^{*}(n)^{2} \gg x(\log \log x)^{3} .
$$

This completes the proof. 


\section{Proof of Theorem 1.2}

We have shown

$$
\sum_{n \leq x} \omega^{*}(n)^{2}=\sum_{[p-1, q-1] \leq x} \frac{x}{[p-1, q-1]}+\mathbf{O}(x) .
$$

Letting $S_{t}$ be the number of solutions of primes $p, q$ with $[p-1, q-1]=t$, our sum becomes

$$
x \sum_{n \leq x} \omega^{*}(n)^{2}=\sum_{t=1}^{x} \frac{S_{t}}{t} .
$$

We make a dyadic subdivision,

$$
x \sum_{k=1}^{\log x} \frac{1}{2^{k}} \sum_{2^{k} \leq t<2^{k+1}} S_{t} .
$$

By Lemma 2.2, the inner sum is $\mathbf{O}\left(2^{k}\right)$ and so the total sum is $(x \log x)$. This completes the proof.

\section{Concluding remarks}

The method clearly extends to the study of the cognate function which counts the number of primes $p$ such that $p-a$ divides $n$ for a fixed value of $n$. Certainly, the lower bound estimate recorded in Theorem 1.1 is immediate in this case also. For the upper bound estimate, one would need the analog of the Erdös-Prachar theorem. Here again, the method extends with little difficulty and we have an analogous theorem in this case as well.

It is interesting to note that if we used Lemma 2.4 in (4.10), we would have obtained a weaker estimate of $\mathbf{O}\left(x(\log x)(\log \log x)^{2}\right)$ in Theorem 1.2.

We have shown above that

$$
\sum_{n \leq x} \omega^{*}(n)^{2}=\sum_{[p-1, q-1] \leq x} \frac{x}{[p-1, q-1]}+\mathbf{O}(x) .
$$

Thus, conjecture (1.3) reduces to the problem of finding an asymptotic formula for the sum on the right hand side. In other words, (1.3) reduces to the alluring conjecture that for some constant $C>0$, as $x \rightarrow \infty$,

$$
\sum_{[p-1, q-1] \leq x} \frac{1}{[p-1, q-1]} \sim C \log x,
$$

where the summation is over primes $p, q \leq x$. We also find it amusing that had the authors of [EP55] cleaned up their paper, they would have seen some sixty-five years earlier, our Theorem 1.2 which improves upon Prachar's 1955 theorem in [Pra55]!

Acknowledgements. We thank the referee and Michel Waldschmidt for helpful corrections to an earlier draft.

\section{References}

[APR83] L. Adleman, C. Pomerance, and R. Rumely, On distinguishing prime numbers from composite numbers, Annals of Math. 117 (1983), 173-206.

[Erd48] P. Erdös, Some asymptotic formulas in number theory, J. Indian Math. Soc. 12 (1948), 75-78.

[EP55] P. Erdös and K. Prachar, Über die Anzahl der Lösungen von $[p-1, q-1] \leq x$, Monatsh. Math. 59 (1955), 318-319. 
[Hal74] H. Halberstam and H.E. Richert, Sieve Methods, Academic Press, 1974.

[HR1917] G.H. Hardy and S. Ramanujan, The normal number of prime factors of a number n, Quart. J. Math. 48 (1917), 76-92.

[MV73] H. Montgomery and R. Vaughan, The large sieve, Mathematika 20 (1973), 119-134.

[Ram08] M. Ram Murty, Problems in Analytic Number Theory, 2nd edition, Springer, 2008.

[MM79] M. Ram Murty and V. Kumar Murty, Some results in number theory I, Acta Arith. 35 (1979), 367-371.

[MM84] M. Ram Murty and V. Kumar Murty, Groups of square-free order, Math. Ann. 267 (1984), $299-309$.

[Nor76] K.K. Norton, On the number of restricted prime factors of an integer, I, Illinois Journal of Math. 20 (1976), $681-705$.

[Pom77] C. Pomerance, On the distribution of amicable numbers, J. reine angew Math. 293/294 (1977), $217-222$.

[Pra55] K. Prachar, Über die Anzahl der Teiler einer natürlichen Zahl, welche die Form $p$ - 1 haben, Monatsh. Math 59 (1955), 91-97.

[Ra1915] S. Ramanujan, Highly composite numbers, Proceedings of the London Math. Society 14 (2), (1915), $347-409$.

[Ra1916] S. Ramanujan, Some formulae in the analytic theory of numbers, Messenger of Mathematics 45 (1916), 81-84.

\section{Ram Murty}

Department of Mathematics and Statistics

Queen's University, Kingston

Ontario, Canada, K7L 3N6

e-mail: murty@queensu.ca

\section{Kumar Murty}

Department of Mathematics

University of Toronto, Toronto

Ontario, Canada, M5S 2E4

e-mail:murty@math.toronto.edu 